\title{
Detection of Proportional Scintillation in Liquid Xenon
}

\author{
E. Aprile ${ }^{a}$, R. Budnik ${ }^{a}$, H. Contreras ${ }^{a}$, L.W. Goetzke ${ }^{a}$, R. Hood ${ }^{a}$, \\ A.J. Melgarejo Fernandez ${ }^{a}$, M. Messina ${ }^{a}$, J. Naganoma ${ }^{* b}$, G. Plante ${ }^{a}$, A. Rizzo ${ }^{a}$, \\ P. Shagin ${ }^{b}$, and R. Wall ${ }^{b}$ \\ ${ }^{a}$ Physics Department, Columbia University, New York, NY 10027, USA \\ ${ }^{b}$ Department of Physics and Astronomy, Rice University, Houston, TX 77005, USA \\ E-mail: junjierice.edu
}

\begin{abstract}
Measurements of the properties of proportional scintillation light emitted in liquid xenon around thin wires are reported. The maximum proportional scintillation gain of $287_{-75}^{+97}$ photons per drift electron was obtained using $10 \mu \mathrm{m}$ diameter gold plated tungsten wire. The threshold for electron multiplication and proportional scintillation is measured as $725_{-139}^{+48}$ and $412_{-133}^{+10} \mathrm{kV} / \mathrm{cm}$, respectively. The threshold for proportional scintillation is in good agreement with previously published result, while the electron multiplication threshold represents a novel measurement. A complete set of parameters for the practical use of the electron multiplication and proportional scintillation processes in liquid xenon was also obtained for the first time. The work was triggered by the promising application of proportional scintillation in liquid xenon for simpler and more sensitive dark matter detectors.
\end{abstract}

Technology and Instrumentation in Particle Physics 2014,

2-6 June, 2014

Amsterdam, the Netherlands

${ }^{*}$ Speaker. 


\section{Introduction}

The search for direct evidence of dark matter interacting with terrestrial particles has been an intense area of research within the worldwide scientific community. A very promising candidate for galactic dark matter is the weakly-interacting massive particle (WIMP), which calculations have shown would have approximately the right relic abundance, assuming a mass and interaction cross section on the GeV scale. Gas-liquid dual-phase time projection chambers (TPC) using noble elements, xenon in particular, have emerged as leaders in this exciting field, and have set the strongest limits to-date on the WIMP-nucleon scattering cross section for a wide range of masses [四, ㅁ]. Liquid xenon (LXe) is an attractive target for studying WIMP interactions with normal matter because it is relatively free of radioactive isotopes, and its physical and chemical properties make it ideal for the production and propagation of ionization electrons and scintillation photons produced by radiation.

Dual-phase TPCs rely on the simultaneous detection of direct scintillation photons produced in the liquid phase (the S1 signal) and proportional scintillation photons (the S2 signal) produced in the gas phase, following extraction of ionization electrons from the liquid to the gas. From the time difference between the S1 and S2 signals and the localized position of the S2 signal, one can infer the location of the initial interaction, allowing for a fiducialization of the detector volume to take advantage of LXe's excellent stopping power for background particles. Furthermore, the ratio of the size of the $\mathrm{S} 1$ and $\mathrm{S} 2$ signals is substantially different for nuclear and electronic recoils, allowing for discrimination between WIMP events and most major backgrounds. These two background rejection methods have been successfully used to make xenon TPC's among the best low background detectors for the measurement of rare nuclear recoils.

An increase in sensitivity beyond that enabled by current experiments ([W, [2]) requires new detectors with a total target mass at least an order of magnitude larger than the current state-ofthe-art. A simple scale-up of a dual-phase TPC presents practical challenges and difficulties, most notably associated with high voltage handling and precise control of liquid-gas interface level, since both the drift gap in the liquid and the active area increase. These challenges are much reduced in a single-phase LXe TPC, as such a detector allows for the implementation of two or more drift gaps with a subsequently reduced high voltage requirement, and does not require a liquid-gas interface. Without total internal reflections suffered by the primary light at the liquid-gas interface, a single phase TPC will also have a higher intrinsic light collection efficiency for the S1 signal [3]].

On the other hand, proportional scintillation in LXe is possible and was indeed observed for the first time by Lansiart et al. [团] in 1976 using thin wires, and confirmed a few years later by Masuda et al. [[]]. We therefore embarked in a study to reproduce and further understand the phenomenon in view of its potential application for a next generation, large scale LXe as the ultimate dark matter detector.

\section{Experimental Setup}

The detector developed for this study is a single-phase TPC, built with four grid electrodes supported by a polytetrafluoroethylene (PTFE) structure, and viewed by two photo-multiplier tubes (PMTs), all immersed in LXe, as shown in figure $\square$ left. The TPC was irradiated with $\alpha$-particles 
emitted by a source deposited on the cathode grid, producing direct scintillation light (the $\mathrm{S} 1$ signal) and ionization electrons. The electric field produced between the cathode and first grid electrode drifts the electrons towards the anode wire, where a strong field accelerates them, leading to the S2 signal in LXe. A charge-sensitive preamplifier was coupled to the anode wire to directly measure the number of electrons produced in the interaction. The TPC was cooled by a cold finger immersed in liquid nitrogen $\left(\mathrm{LN}_{2}\right)$. Once filled in the detector, the xenon was continuously purified using a high temperature getter, throughout the duration of each experiment. A schematic drawing of the TPC, and pictures of the experimenal setup, including the cooling and purification systems are shown in figure $\mathbb{~}$. The setup was located at Columbia University's Nevis Laboratories.
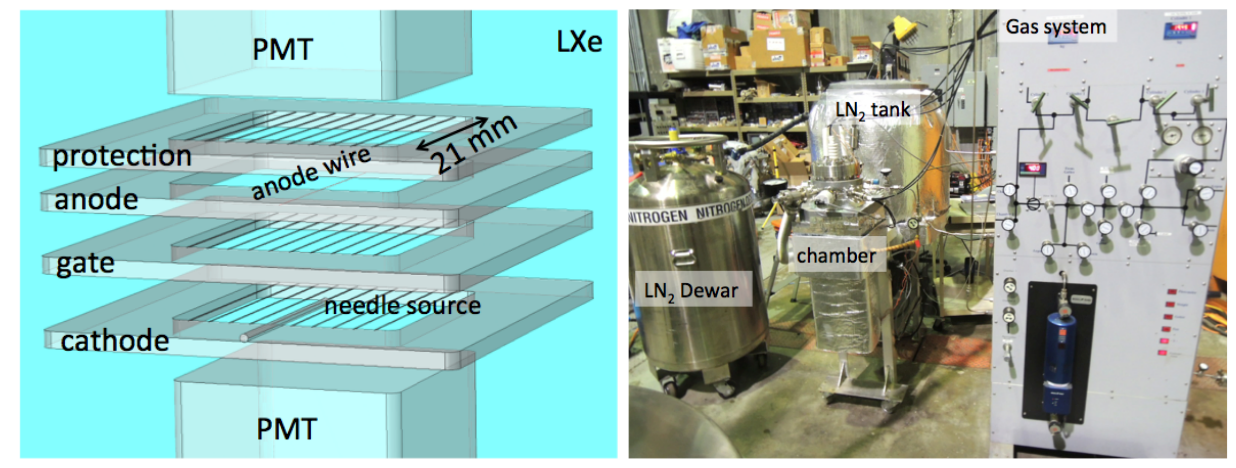

Figure 1: Left: schematic drawing of the single phase LXe TPC. A thin wire is placed on the anode frame, and a needle $\alpha$ source is placed on the cathode frame. Right: The complete experimental setup, including the cooling and gas circulation and purity control systems.

\section{Analysis}

Recorded digitized waveforms for the top and bottom PMTs, together with the charge signal, were analyzed to study the properties of proportional scintillation in LXe. Event selection criteria were applied to the data in order to reject background events coming from cosmic ray interactions and other environmental sources, and to select good events coming from $\alpha$-particle interactions in the LXe. In order to fully characterize the proportional scintillation process, a number of quantities must also be derived using simulations. For example, to determine the number of scintillation photons produced in the interaction, it is necessary to calculate the S2 light collection efficiency for the top and bottom PMTs. The experimental light collection efficiencies, the precise electric field map, and the expected electron drift time distribution were studied in detail using COMSOL and Geant4.

\subsection{Simulation}

The COMSOL multi-physics simulation package was used to model the electric field inside the detector for a variety of voltage configurations. The result of an electric field simulation in the drift region and the area close to the anode wire is shown in figure 2 . Three possible drift paths are clearly visible, two of which have equal drift length. A small misalignment of the needle source 
with respect to the other electrodes breaks the symmetry of the two longer drift paths shown in figure 2 , and result in three distinct drift paths. Only the central path was chosen to minimize the effect of electrode misalignment.
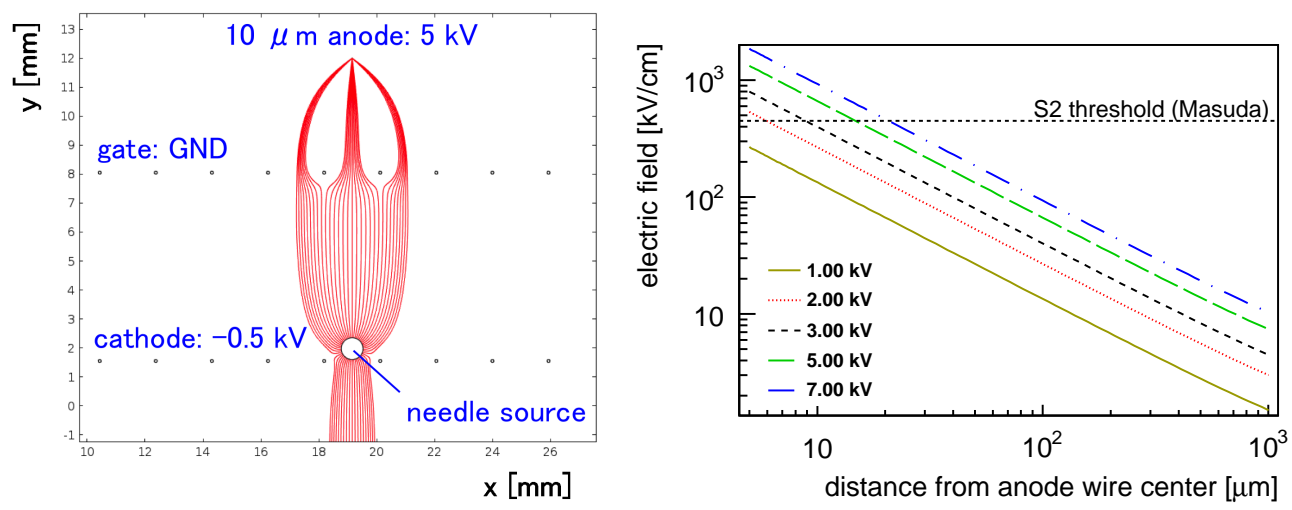

Figure 2: Left: The result of the electric field simulation for the drift region. Charge drift streamlines from the needle source are shown in red. Three main drift paths towards the anode wire are clearly visible. No streamlines are present which end at the gate electrode wires. Right: Value of the electrical field as a function of the distance from the anode wire center (10 $\mu \mathrm{m}$ diameter wire). Solid, dotted, dashed, long-dashed and long-dashed-dotted lines correspond to voltage between the gate and the anode $\left(V_{A}\right)=1,2,3,5$, and $7 \mathrm{kV}$, respectively. A horizontal dashed line represents the S2 threshold from [ [D].

To obtain the absolute number of emitted S2 photons, a dedicated simulation was used to estimate the light collection efficiency (LCE) of the two PMTs. The Geant4 toolkit [G] was used to model the LCE for both top and bottom PMTs in a small region just below the anode wire. The simulated LCE for the top and bottom PMTs is shown in figure B. The LCE for the bottom PMT is approximately $21 \%$ and mostly independent of the distance from the anode wire. Shadowing of the scintillation light by the anode wire itself leads to the observed drop in LCE for the top PMT in the region closest to the anode wire. For these reasons, the $\mathrm{S} 2$ signal measured by the bottom PMT was used for the data analysis.

\subsection{Event selections}

Several selections were applied to the PMT signals to reject different sources of background (cosmic ray, environmental, etc.) and to reject the events with lower quality originating from the $\alpha$-particle interaction. First, the size of the S1 signal must be compatible with the expected signal size from an $\alpha$-particle interaction in the LXe. Further, events must show more of the S1 signal on the top PMT than the bottom one, in order to reject drift paths from the sides of the anode wire. Second, the pulse height of the $\mathrm{S} 2$ signal must be more than $2 \mathrm{mV}$ to reject electronic noise and/or thermal electron emission from the PMT photocathode. Third, the time difference between the S1 and the S2 signals (termed the drift time) must be consistent with the drift distance between the anode and the cathode. Last, the S2 signal time width must be consistent with the lone central drift path (see figure $\square$ ) to reject events in which other drift paths are used. While no significant change in the counting rates is seen for $V_{A}>1.25 \mathrm{kV}$, the event selection rate drops at lower voltages due to a smaller average $\mathrm{S} 2$ signal size. 


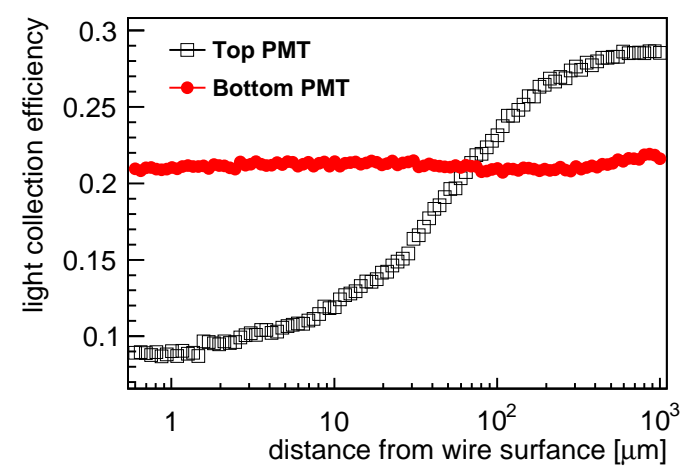

Figure 3: LCE of the S2 signal for the top (squares) and bottom (circles) PMTs as a function of the distance from the anode wire surface (10 $\mu \mathrm{m}$ diameter wire). While LCE for the bottom PMT is almost independent of the distance to the anode surface, the one for the top PMT decreases near the anode due to the shadowing of the scintillation light by the anode wire surface itself.

The bottom PMT S2 distribution and the averaged charge signal after application of the event selection criteria are shown in figure 6 . Preamplifier waveforms were averaged over many events to improve the signal to noise ratio.
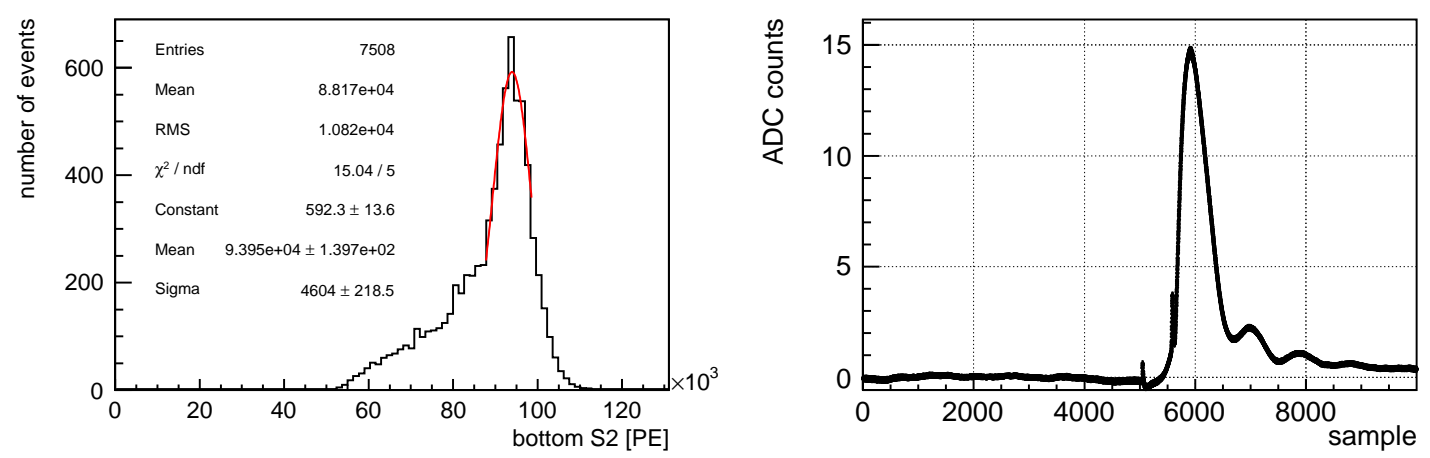

Figure 4: A typical result obtained using the $10 \mu \mathrm{m}$ diameter anode wire with $V_{A}=5.00 \mathrm{kV}$. Left: The S2 signal distribution after the event selection criteria are applied. The peak of this distribution is fit with a Gaussian to define its mean and width. Right: Averaged charge signal after the event selection. The small peaks seen at 5000 and 5600 samples are due to pick up noise induced by the S1 and S2 signals in the PMTs.

\section{Results}

In this section, the main analysis results are presented. First, the main sources of systematic uncertainty are discussed. Analysis of the 10 and $5 \mu \mathrm{m}$ data follow, in which the threshold for proportional scintillation and charge multiplication are extracted. Finally, results are presented related to the S2 signal width and resolution, with a discussion on the proportionality of the observed S2 signal. 


\subsection{Systematic uncertainties}

Two main classes of systematic uncertainty on the size of the S2 signal were estimated. The first class comprises those uncertainties which in principle depend on the high voltage conditions of the experiment, specifically $V_{A}$. Total relative uncertainties on this type for the $10 \mu \mathrm{m}$ anode wire are approximately $15 \%$ (10\%) for the PMT (preamplifier) signals. Both uncertainties are dominated by the stability over time. Those uncertainties for the data with a $5 \mu$ m anode wire are $6 \%$ and $20 \%$.

The other major class of systematic uncertainties affecting the analysis is related to the factor $(g)$ used to convert the S2 gain in photo-electrons to the S2 gain in photons per drift electron $\left(p h / e^{-}\right)$. Unlike the uncertainties discussed above, this factor does not depend on $V_{A}$. This conversion factor is defined as

$$
g=\frac{S 2}{\frac{E_{\alpha}}{W} f_{i o n} \varepsilon_{L C} \varepsilon_{Q} f_{L X e} \varepsilon_{d y}},
$$

where $E_{\alpha}$ is the energy of $\alpha$-particles emitted by ${ }^{210} \mathrm{Po}, W$ is the ionization energy, $f_{\text {ion }}$ is the fraction of electrons which escape recombination [ $\left[\right.$ ],$\varepsilon_{L C}$ is the bottom PMT S2 LCE, $\varepsilon_{Q}$ is the QE of the PMTs at ambient temperature, $f_{L X e}$ is a correction factor for the $\mathrm{QE}$ to account for the difference between ambient and LXe temperature, and $\varepsilon_{d y}$ is the photo-electron collection efficiency at the first dynode. The uncertainty on $f_{\text {ion }}$ comes from the electric field variation around the needle source, which can vary from 1.5 to $3.0 \mathrm{kV} / \mathrm{cm}$. This uncertainty is estimated by electric field simulation and is dominated by the leakage potential from the anode wire through the gate wires and the geometric effect of the wire's round shape. Those uncertainties are about $30 \%$.

\subsection{Threshold extraction and other results}

The primary result from the acquired data is the evolution of the proportional scintillation signal in LXe as a function of the voltage on the anode wire. Figure $\square$ shows the relative charge signal from the preamplifier (left), and the S2 signal from the bottom PMT (right) measured with the 10 and $5 \mu \mathrm{m}$ anode wires. A maximum average S2 signal of $(2.28 \pm 0.34) \times 10^{5}$ PEs was obtained with the $10 \mu \mathrm{m}$ anode wire at $V_{A}=6.75 \mathrm{kV}$. This corresponds to an $\mathrm{S} 2$ gain of $287_{-75}^{+97} p h / e^{-}$, with a factor of $\sim 14$ electron multiplication.

To extract the physical properties of electron multiplication and proportional scintillation in LXe, expressions derived for xenon in the gas phase in [ [ 8, Q] were modified to remove the pressure dependence and were used to fit the charge and S2 signals in LXe:

$$
\begin{aligned}
\Delta N_{e} & =N_{e} p_{0} \exp \left(-\frac{p_{1}}{E-p_{2}}\right) \Delta L \\
\Delta N_{\gamma} & =N_{e} p_{3}\left(E-p_{4}\right) \Delta L
\end{aligned}
$$

where $N_{e}$ is the number of electrons, $p_{i}$ are the fit parameters, $E$ is the electric field strength, $\Delta L$ is the drift length of the electron, and $N_{\gamma}$ is the number of S2 photons. The charge and S2 signal data points are fitted simultaneously as $N_{e}$ increases as the electrons drift. The electric field strength at each point was obtained from COMSOL simulation. Table $\square$ shows the results for the simultaneous as well as individual fits to the 10 and 5 um data samples. The main parameters of interest are $p_{2}$ 

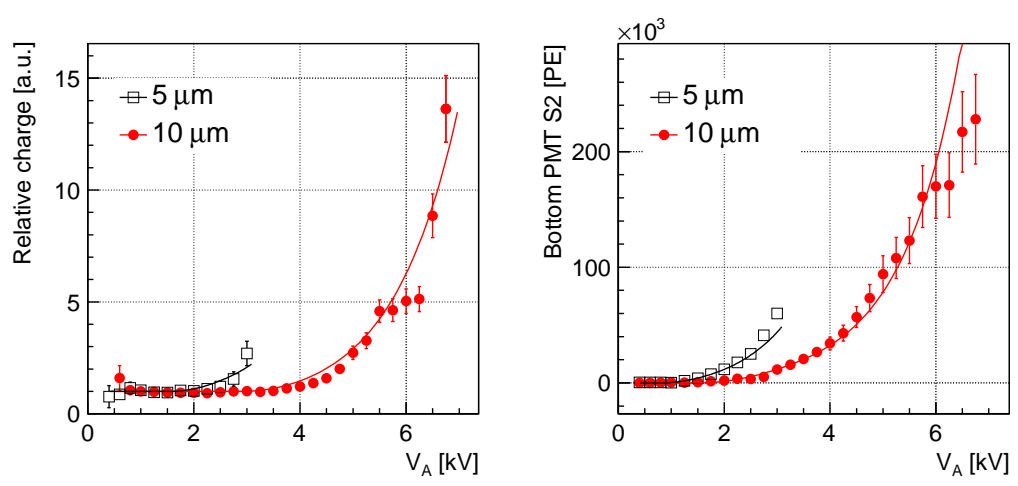

Figure 5: Left: The preamplifier relative charge signal as a function of $V_{A}$. While the amount of charge is flat at lower voltages, it rapidly increases at higher voltages, indicating the onset of charge multiplication. Right: The bottom PMT S2 as a function of $V_{A}$. The lines are from the simultaneous fit of the $10 \mu \mathrm{m}$ and $5 \mu \mathrm{m}$ data points for both charge and $\mathrm{S} 2$.

(the threshold for charge multiplication), $p_{3}$ (the $\mathrm{S} 2$ gain factor in $\mathrm{PE} /(\mathrm{kV} / \mathrm{cm} \cdot \mu \mathrm{m})$ ), and $p_{4}$ (the threshold for $\mathrm{S} 2$ production). The quoted $\mathrm{S} 2$ gain (in $p h / e^{-} /(\mathrm{kV} / \mathrm{cm} \cdot \mu \mathrm{m})$ ) is calculated using $p_{3}$ and the correction factor $g$ defined in the previous section.

Positive correlations between the gain and threshold were obtained since the threshold at higher electric field requires a higher gain. While the fits describe the overall features of electron multiplication and proportional scintillation, some discrepancy does remain between the data and this simple model. In particular, while the threshold of proportional scintillation is consistent for all the fits, other parameters are not consistent.

Considering the disagreement of the results from different fits as uncertainties, the thresholds of electron multiplication and proportional scintillation are inferred to be $725_{-139}^{+48}$ and $412_{-133}^{+10} \mathrm{kV} / \mathrm{cm}$, respectively. The central value was obtained from the 10 and $5 \mu \mathrm{m}$ simultaneous fit. The thresholds correspond to the $\mathrm{S} 2$ proportional signal starting at $\sim 10 \mu \mathrm{m}$ from the anode wire surface and electron multiplication starting at a few $\mu \mathrm{m}$ from the anode wire surface.

Table 1: Results from a simultaneous fit of the 10 and $5 \mu \mathrm{m}$ data and individual fits of the 10 and $5 \mu \mathrm{m}$ data. The last line shows the $\mathrm{S} 2$ gain factor after converting PE to $p h / e^{-}$.

\begin{tabular}{lrrr}
\hline \hline parameter & $10 \& 5 \mu \mathrm{m}$ & only $10 \mu \mathrm{m}$ & only $5 \mu \mathrm{m}$ \\
\hline$p_{0}:$ charge gain factor $\left[1 /\left(\mu \mathrm{m} \cdot e^{-}\right)\right]$ & $0.80 \pm 0.10$ & $1.15 \pm 0.15$ & $1.46 \pm 0.02$ \\
$p_{1}:$ slope in charge gain $[\mathrm{kV} / \mathrm{cm}]$ & $242 \pm 45$ & $561 \pm 12$ & $298 \pm 1$ \\
$p_{2}:$ threshold of charge mult. $[\mathrm{kV} / \mathrm{cm}]$ & $725 \pm 48$ & $586 \pm 47$ & $750 \pm 1$ \\
$p_{3}:$ S2 gain factor $[\mathrm{PE} /(\mathrm{kV} / \mathrm{cm} \cdot \mu \mathrm{m})]$ & $16.6 \pm 1.1$ & $13.3 \pm 0.4$ & $17.9 \pm 3.4$ \\
$p_{4}:$ threshold of S2 $[\mathrm{kV} / \mathrm{cm}]$ & $412 \pm 10$ & $399 \pm 7$ & $416 \pm 13$ \\
$\chi^{2} / \mathrm{ndf}$ & $125 / 63$ & $71.4 / 42$ & $19.9 / 16$ \\
\hline$p_{3}: \mathrm{S} 2$ gain factor $\left[\mathrm{ph} / \mathrm{e}^{-} /(\mathrm{kV} / \mathrm{cm} \cdot \mu \mathrm{m})\right]$ & $\left(2.09_{-0.47}^{+0.65}\right) \times 10^{-2}$ & $\left(1.68_{-0.36}^{+0.51}\right) \times 10^{-2}$ & $\left(2.26_{-0.65}^{+0.80}\right) \times 10^{-2}$ \\
\hline \hline
\end{tabular}


Proportional scintillation was observed at $V_{A}$ even below the $\mathrm{S} 2$ threshold. The fit disagrees significantly with the data at lower $V_{A}$ as shown in figure (left). The $\mathrm{S} 2$ width at $10 \%$ pulse height decreases at these voltages as shown in figure 6 (right). An S2 width of $300 \mathrm{~ns}$ is consistent with the expectation that the electron cloud is spread by longitudinal and transverse diffusions [ए]]. The $\mathrm{S} 2$ width reduction at lower $V_{A}$ indicates that only some fraction of electrons acquire energy by the electric field and emit S2 light, and therefore the actual threshold is not determined by the electric field.
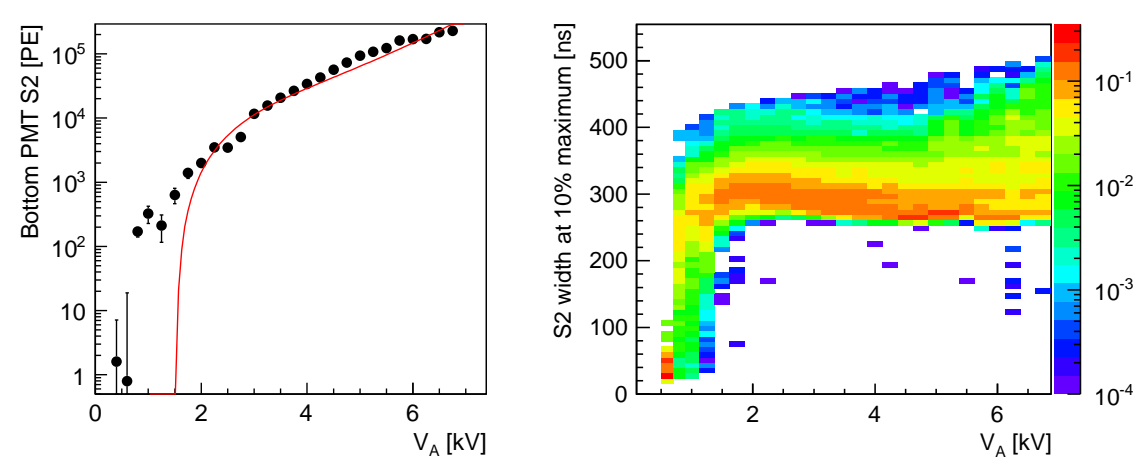

Figure 6: Left: $\mathrm{S} 2$ as a function of $V_{A}$ from the $10 \mu \mathrm{m}$ anode wire in log scale. Significant disagreement between data and the fit can be seen. Right: S2 pulse width at $10 \%$ of pulse height. The colors represent number of events normalized at each $V_{A}$. The $\mathrm{S} 2$ width decreases at low voltages.

S2 signals comprised of less than 10 electrons are important for the study of WIMPs with a mass down to a few $\mathrm{GeV}$. The observed maximum S2 gain of $287_{-75}^{+97}$ photons per electron from this study indicates that it is possible to detect a signal from a few drift electrons. Even without further optimization, this implies the possibility to utilize proportional scintillation with thin wires in LXe for next generation dark matter detectors, though it is important to note that other applications are possible.

\section{References}

[1] E. Aprile, et al. (XENON100 Collaboration), Phys. Rev. Lett. 109 (2012)181301.

[2] D. S. Akerib, et al. (LUX Collaboration), Phys. Rev. Lett. 112 (2014) 091303.

[3] Private comminication with K. Giboni. The idea can be found at KEK seminar.

[4] A. Lansiart, et al., Nucl. Instrum. Methods 135 (1976) 47.

[5] K. Masuda, et al., Nucl. Instrum. Methods 160(1979) 247.

[6] S. Agostinelli, et al., Nucl. Instrum. Methods A506 (2003) 250.

[7] E. Aprile, et al., Nucl. Instrum. Methods A307(1991) 119.

[8] W.R. Leo, Techniques for Nuclear and Particle Physics Experiments, Springer-Verlag (1993).

[9] A.I. Bolozdynya, Nucl. Instrum. Methods A422 (1999) 314.

[10] E. Aprile, and T. Doke, Rev. Mod. Phys. 82 (2010) 2053. 